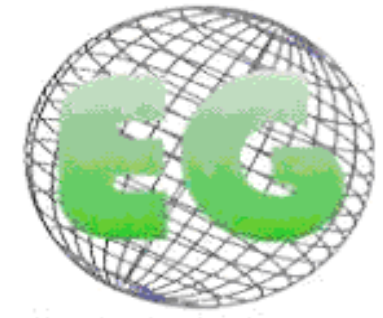

ISSN 1695-6141 N'25

\title{
Conocimiento de los padres en la prevención del abuso sexual infantil
}

Parental Knowledge in the prevention of child sexual abuse

\author{
*Morillo, B; *Montero, L.; *Colmenares, Z. \\ *Magíster en Enfermería Materno Infantil Mención Obstetricia. Docente de la Escuela de Enfermería Dra. \\ Gladys Román de Cisneros. Universidad de Carabobo. Venezuela.
}

Palabras clave: abuso infantil; abuso sexual infantil; prevención.

Keywords: child abuse; child sexual abuse; prevention

\section{RESUMEN}

El abuso sexual infantil se presenta mayormente a nivel intrafamiliar es decir son los padres, tíos, hermanos, padrastros, que se aprovechan de la inocencia del niño, situación esta que impacta en gran manera su vida; el presente estudio tuvo como propósito Determinar el Nivel de conocimiento que poseen los padres en Relación al Abuso Sexual Infantil. Se efectuó un estudio de campo de carácter descriptivo, no experimental transeccional. La población considerada estuvo conformada por 80 madres de los niños y niñas en edad escolar del sector Colinas de Mara II, Morón, estado Carabobo. La muestra correspondió $25 \%$ de la población objeto del estudio es decir 19 madres. La recolección de la información se obtuvo a través de un cuestionario con 19 ítems, de alternativas de respuestas dicotómicas (Sí-NO), siendo validado por juicio de expertos. Y un coeficiente de confialidad de 0,79 por el método de alfa de Crombach. Del Análisis de los resultados se Concluyó, que un $50 \%$ de los padres encuestados no dialogan con su hijos sobre esta situación, medida vital que permite prevenir el abuso sexual infantil, lo que hace más relevante la necesidad de poner en práctica estrategias educativas para la prevención del abuso sexual infantil y así contribuir a combatir este flagelo social.

\section{ABSTRACT}

Child sexual abuse occurs mostly within the family, i.e. the parents, uncles, brothers, stepfathers, who prey on the innocence of youth, a situation that impacts greatly on the child's life. This study was aimed at determining the level of knowledge parents have in relation to Child Sexual Abuse. A cross-sectional, non experimentan descriptive field study was made. The population consisted of 80 mothers of school age children from the Mara Hill II sector, Moron, Carabobo State. The sample consisted of $25 \%$ of the population surveyed, i.e. 19 mothers. Data collection was obtained through a questionnaire with 19 items of dichotomous response options (YESNO), and was validated by expert opinion. The confidence coefficient was 0.79 according to the Crombach alpha. Analysis of the results concluded that $50 \%$ of the parents surveyed do not talk to their children about the situation, when talkimg is a vital method of preventing child sexual abuse. It is, theregore, of great importance implement educational strategies for prevention of child sexual abuse and so help to combat this social scourge. 


\section{INTRODUCCIÓN}

Cuando se habla de abuso infantil podemos decir que los niños, niñas, son las verdaderas víctimas de la sociedad y desde inicios de la civilización han sido objeto de abandono, abuso y de violencia por parte de personas mayores y especialmente de sus padres. ${ }^{(1)}$

El abuso infantil es todo daño producido a un niño de manera accidental, por una persona de mayor edad, más fuerte o autoritaria, sobre la que el niño no tiene control, que produce trastornos físicos y mentales que impiden el desarrollo normal para el desenvolvimiento en la vida. ${ }^{(2)}$ La coerción emocional y física que ejerce el abusador sobre la víctima, tiene como fin garantizar su silencio. ${ }^{(3)}$

Dentro del abuso infantil encontramos el abuso sexual, que consiste en hacer practicar al niño actividades sexuales tales como: tocar o hacer tocar de manera seductiva cualquier parte del cuerpo, no solo los genitales, del niño o del adulto; mirar o hacer mirar las partes íntimas del niño o del adulto, mostrar o tomar fotos explícitamente sexuales, hacer observar al niño actividades sexuales. También puede no haber contacto corporal o visual, como propuestas verbales indecentes, pero que en todos los casos se trata de actividad que el niño por inmadurez no puede comprender y para los cuales no está preparado ni física ni emocionalmente. ${ }^{(2)}$

El Abuso Sexual infantil es la formas más severa de maltrato infantil, además en muchos países constituye un delito penalizado por la ley, pero cuando el abuso es intrafamiliar, raramente se descubre la primera vez que sucede, por lo general transcurren meses o inclusive años hasta que el secreto se desvela ${ }^{(3)}$ y en muchas ocasiones este acto no se comunica a la justicia.

Gran parte de los niños traumatizados sexualmente son víctimas de alguien que conoce y en quien se confía, la mayoría de estas agresiones ocurren dentro del entorno habitual del menor ${ }^{(4)}$ y un alto número de los casos reportados tienen lugar entre los miembros de la extensión familiar: abuelos, tíos, primos y otro parientes de la periferia ${ }^{(5)}$.

En Venezuela y en el mundo entero, innumerables son los factores y consecuencias que propician esas irregularidades. El abuso sexual sobre menores es una realidad patente de la sociedad actual, siendo los niños los principales perjudicados ya que esta acción constituye uno de los traumas psíquicos más intensos y de consecuencias sumamente destructivas para la estructura de la personalidad.

Todo lo precedente evidencia las graves implicaciones para la salud física y mental del niño, niña y adolescente, que acarrea este problema, por los que los profesionales de la salud tienen un papel fundamental en la intervención, detección y prevención del maltrato infantil , por lo que se deben sumar esfuerzos a fin de detectar e interrumpir el abuso de la manera más precoz posible, ya que el diagnostico del niño mejora cuando menor haya sido el tiempo de exposición, aunque es muy difícil calibrar la magnitud del daño a largo plazo puesto que los episodios traumáticos impactan ${ }^{(3)}$

Por todo lo anteriormente expuesto se ejecutó un trabajo de investigación cuyo objetivo general fue Determinar el nivel de conocimiento que poseen los padres en relación al abuso infantil, en el Municipio Juan José Mora, de Puerto Cabello, estado Carabobo, el cual va a permitir a los padres un enfoque con otra visión, en relación con el significado de la educación sexual y de esta manera orienten a temprana edad a sus hijos, sobre la 
prevención del abuso sexual, y establezcan una comunicación efectiva la cual de vital importancia como método de prevención del abuso sexual Infantil.

\section{MATERIALES Y MÉTODOS}

Se utilizó para el estudio una metodología de investigación cuantitativa, con un diseño No experimental, de campo, descriptivo; La Población objeto de estudio estuvo conformada por 80 madre de los niños, niñas y adolescentes del Sector la Colinas, Municipio Juan José Mora, de Puerto Cabello, estado Carabobo. La muestra probabilística al azar simple de 19 madres que representó el $20 \%$ de la población. Se recolectó la información a través de un instrumento, tipo cuestionario con alternativas de respuestas dicotómicas, contó con 19 ítems. La validación del instrumento se realizó mediante la aplicación de los siguientes procedimientos: validación por juicio de expertos en contenido, tomando en cuenta el tipo de estudio, objetivos y operacionalización de la variable. Se realizó prueba piloto con un índice de confiabilidad de 0,79 mediante el alfa de Crombach. Los datos se analizaron y se transfirieron a una matriz de datos, luego se tabuló en cuadro y gráficos.

\section{ANÁLISIS DE RESULTADOS}

Gráfico 1.- Representación de la distribución de la opinión de los encuestados en relación al Abuso Sexual dentro del Hogar
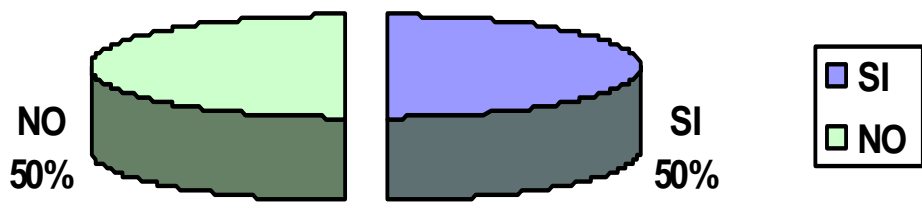

Fuente: Instrumento aplicado, abril 2009

Los resultados obtenidos evidenciaron en un 50\% que los padres afirman que frecuentemente el autor del abuso sexual infantil proviene del entorno social externo de los niños, niñas , mientras que el otro $50 \%$ negó que esta situación pueda ocurrir en el entorno familiar, situación esta que evidencia la confianza que el grupo de padres muestra hacia entorno familiar y poner en situaciones de riesgo al infante por este exceso de confianza. Ya que piensan que esto no pudiese pasar con un familiar. 
Gráfico 2.- Representación de la distribución de la opinión de los encuestados en relación a la Interferencia en el Desarrollo de Niños y Niñas.

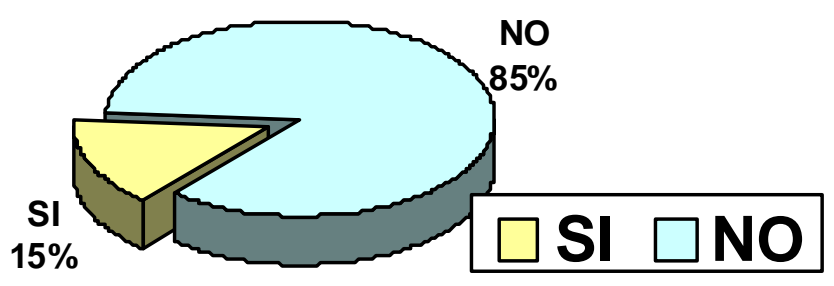

Fuente: Instrumento aplicado, abril 2009

$15 \%$ de los padres respondió que el abuso sexual infantil interfiere en el desarrollo psicológico del niño, niña, mientras que un $85 \%$ opinó que esta situación no afecta la esfera psíquica del infante. Situación si se quiere alarmante ya que no están conscientes del impacto que esta situación acarrea en la vida de este ser, ya que el abuso sexual infantil constituye uno de los traumas psíquicos más intensos y de consecuencias sumamente destructivas para la estructura de la personalidad del niño.

Gráfico 3- Representación de la distribución de la opinión de los encuestados en relación al Diálogo sobre el Abuso Sexual

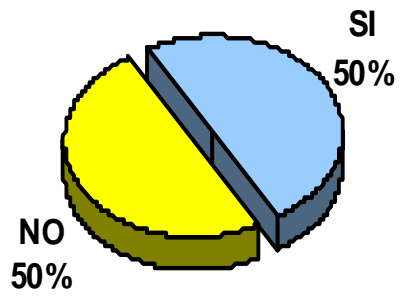

Fuente: Instrumento aplicado, abril 2009

De acuerdo a los resultados obtenidos un 50\% respondió que dialogan con sus hijos sobre el abuso sexual infantil y las situaciones que son consideradas de riesgo, mientras que el $50 \%$ restante expresó que no conversan esta situación que representan riesgo para sus hijos, siendo relevante el hecho de que la comunicación es la base fundamental para evitar 
este tipo de problemas dentro y fuera del núcleo familiar ya que permite al niño comunicar a sus padres sobre alguna situación que pudiera ponerlo en riesgo y alertar al grupo familiar.

Gráfico 4.- Representación de la distribución de la opinión de los encuestados en relación a la Exploración del Cuerpo de Niños y Niñas

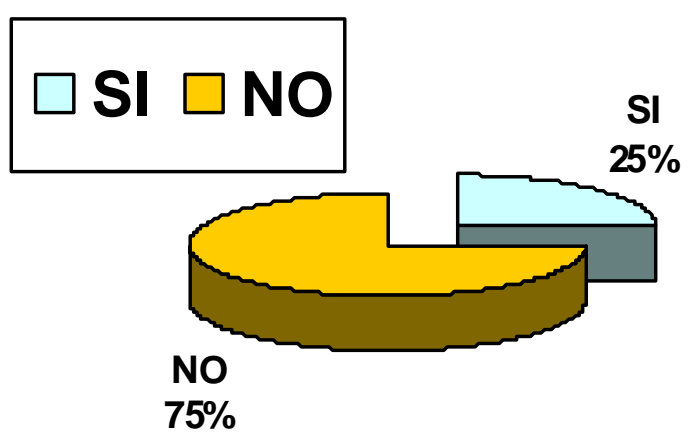

Fuente: Instrumento aplicado, abril 2009

Es importante señalar que $75 \%$ de los padres opinó que los niños y niñas no perciben de manera instintiva dónde empieza la explotación de su cuerpo, mientras que el $25 \%$ respondió que sí. Esta situación queda evidenciada por la respuesta presentada en el gráfico número 3 , en cuanto al déficit de comunicación que existe entre padres e hijo, por los niños y niñas no tienen conocimiento de las situaciones de riesgos de abuso sexual infantil e informarlas oportunamente a sus padres y prevenir que sean víctimas de este.

\section{CONCLUSIONES Y RECOMENDACIONES}

\section{Conclusiones}

Se pudo identificar las causas que predisponen a una persona a cometer abuso sexual en los niños, con las consecuencias en los niños para el resto de su vida, interfiriendo su bienestar social y psicológico.

- En el análisis de los resultados un porcentaje de la población conocen las situaciones en que sucede el abuso infantil. Sin embargo, por miedo, o quizás el tabú, los impide en cierta forma, hacer algo al respecto, en defensa de sus propios hijos, por tanto, esta investigación es una ventana hacia una mejor orientación, y conocimientos de sus derechos, siendo un respaldo para aquellos padres y madres de familias que se encuentren en estas situaciones. 
- De acuerdo a los resultados obtenidos los padres respondieron en un 50\% que no dialogan con sus hijos sobre el abuso sexual infantil y las situaciones que son consideradas de riesgo.

- Es importante señalar que $75 \%$ de los padres, opinó que los niños y niñas no perciben de manera instintiva dónde empieza la explotación de su cuerpo,. Se observó en el estudio déficit de comunicación que existe entre padres e hijo, por los niños y niñas no tienen conocimiento de las situaciones de riesgos de abuso sexual infantil e informarlas oportunamente a sus padres y prevenir que sean víctimas de este.

- Los padres entrevistados opinaron en un $85 \%$ que el abuso sexual infantil no interfiere en el desarrollo psicológico del niño, niña.

\section{Recomendaciones}

- Desarrollar programas educativos encaminados a prestar ayuda a la familia, en aras de mejorar la comunicación entre padres e hijos y por ende la protegerlos de este flagelo.

- Educar a la población y capacitar al equipo de salud y sobre este fenómeno, para desarrollar una red multidisciplinaria de protección en toda la comunidad, con actividades que contribuyan a evitar y/o disminuir la incidencia de este flagelo.

\section{A los Padres}

- Mantener la comunicación efectiva con sus hijos, la cual les permitirá un clima de seguridad y confianza, que permitirá evitar la exposición a ser víctimas del la violencia sexual infantil.

- Darle atención adecuada a sus hijos y crear conciencia de quienes dejan al cuidado de sus hijos.

\section{A las Instituciones Educativas y de Salud:}

- Realizar las denuncias respectivas al momento de recibir a niños y niñas con signo de abuso sexual.

- Capacitar al equipo de salud y de los docentes sobre el marco legal sobre la protección infantil existente en el país. 


\section{REFERENCIAS BIBLIOGRÁFICAS}

1.-Casado j.; Díaz J. y Martínez C. (1997) El Niño Maltratado, Ediciones Díaz de los Santos, S.A., Madrid.

2.- Diner M. (2006) Abuso Infantil La Guía de Prevención y Detección. Publicado por:

Pdaxpert, Sount Horizon I.N.C., Miami, Florida, Capitulo I pag 19.

3- Intebi I.(2008) Violencia, abuso sexual infantil: En la mejores familias. Tercera edición, editorial Granica s.a. Argentina- Capítulo I Pág 41

4.-Suvillan D., y Evertine L. (2004) El Sexo que se Calla. Editorial Pax, México.

5.-Finkelhor D. (2005) Abuso Sexual al menor. Editorial pax. México. 